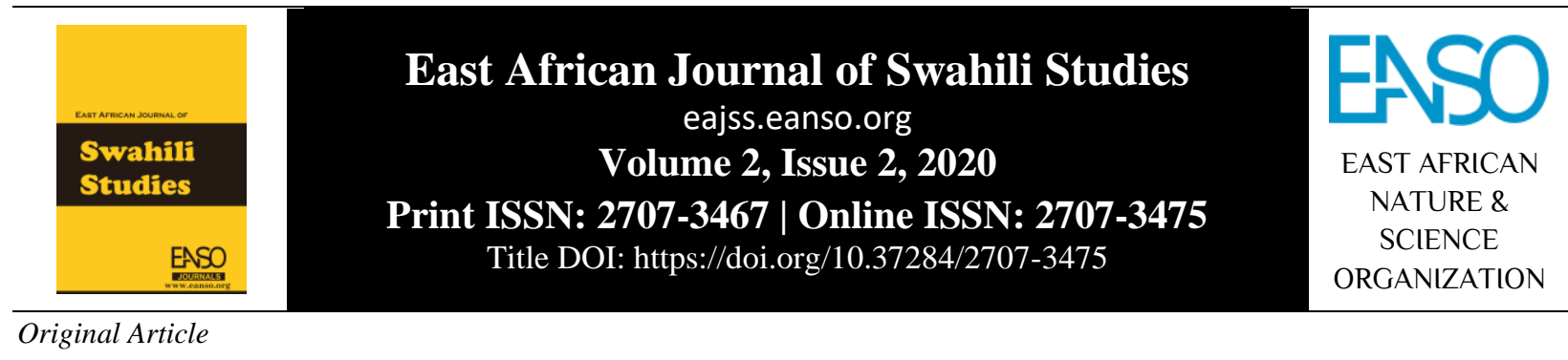

\title{
Matumizi ya Lugha za Kiafrika Kuelekeza Wasichana Katika Masuala ya Ndoa: Mfano wa Matumizi ya Semi Katika Jamii ya Wamasaaba Nchini Uganda
}

\author{
Dr. Willy Wanyenya ${ }^{*}$ \\ ${ }^{1}$ Makerere University Business School, P. O. Box 1337, Kampala, Uganda. \\ *Barua pepe ya mawasiliano: willywanyenya@gmail.com \\ ORCID: https://orcid.org/0000-0002-7594-8256
}

DOI ya Nakala: https://doi.org/10.37284/eajss.2.2.169

Tarehe ya Uchapishaji: IKISIRI

23 June 2020 Katika makala haya, mtafiti anaeleza jinsi lugha inavyotumiwa na wazazi katika shughuli ya kuelekeza mabinti zao katika maisha ya ndoa. Anafanya Maneno Muhimu: hivyo kwa kurejelea ndoa za kijadi katika jamii ya Wamasaaba nchini Uganda. Kwa kurejelea jamii hii, mtafiti anaeleza namna wazazi wanavyotumia lugha Ndoa, kuelekeza mabinti zao katika mambo yanayopelekea ndoa zao kudumu. Semi, Jadi, Elekeza, Lugha Asili, Masuala. Mtafiti alinukuu baadhi ya semi ambazo zinasemekana kuwa zinatumiwa na wazazi katika shughuli hiyo. Ni kweli kwamba lugha yoyote ile huwa inafanya kazi nyingi sana kama vile kutumbuiza watu, kutongozana, kugombana, kufundisha, kuelekeza, n.k. Hii ni kwa sababu bila kutumia lugha, mtu hawezi akaimba wimbo, akagombana, akafundisha, na akaelekeza. Hata hivyo, katika makala hii, mtafiti anajikita kwenye mchango wa lugha katika maisha ya ndoa. Katika kazi hii, mtafiti anazingatia semi mbalimbali ambazo Wamasaaba huzitumia kwa ajili ya kuelekeza mabinti zao katika masuala ya ndoa. Katika jamii mbalimbali, watu hutumia lugha kutekeleza jambo hili. Jamii hasa zile za jadi zilitumia lugha kuelekeza mabinti katika mambo ya ndoa. Kuna namna ambavyo walisuka lugha kisanaa kwa lengo la kufunza mabinti maisha ya ndoa. Hii ni kwa sababu hawakuwa na madarasa maalumu mwa kufunzia watoto wao kama ilivyo sasa. Katika jamii hizo, shule zenyewe hazikuwepo. Kwa hiyo, lugha ndiyo ilikuwa chombo maalumu cha kufunzia watoto. Matumizi ya lugha yalidhamiria kukuza mabinti ambao wangevumilia maisha ya ndoa. Hivyo basi, katika kazi hii, mtafiti anajikita katika matumizi ya lugha na kueleza jinsi Wamasaaba wanavyotumia semi mbalimbali kuelekeza mabinti zao katika masuala ya ndoa. 


\section{APA CITATION}

Wanyenya, W. (2020). Matumizi ya Lugha za Kiafrika Kuelekeza Wasichana Katika Masuala ya Ndoa: Mfano wa Matumizi ya Semi Katika Jamii ya Wamasaaba Nchini Uganda. East African Journal of Swahili Studies, 2(2), 23-31. https://doi.org/10.37284/eajss.2.2.169

\section{CHICAGO CITATION}

Wanyenya, Willy. 2020. "Matumizi ya Lugha za Kiafrika Kuelekeza Wasichana Katika Masuala ya Ndoa: Mfano wa Matumizi ya Semi Katika Jamii ya Wamasaaba Nchini Uganda”. East African Journal of Swahili Studies 2 (2), $23-31$. https://doi.org/10.37284/eajss.2.2.169.

\section{HARVARD CITATION}

Wanyenya, W. (2020) "Matumizi ya Lugha za Kiafrika Kuelekeza Wasichana Katika Masuala ya Ndoa: Mfano wa Matumizi ya Semi Katika Jamii ya Wamasaaba Nchini Uganda”, East African Journal of Swahili Studies, 2(2), pp. 23-31. doi: 10.37284/eajss.2.2.169.

\section{IEEE CITATION}

W. Wanyenya, "Matumizi ya Lugha za Kiafrika Kuelekeza Wasichana Katika Masuala ya Ndoa: Mfano wa Matumizi ya Semi Katika Jamii ya Wamasaaba Nchini Uganda”, EAJSS, vol. 2, no. 2, pp. 23-31, June 2020.

\section{MLA CITATION}

Wanyenya, Willy. "Matumizi ya Lugha za Kiafrika Kuelekeza Wasichana Katika Masuala ya Ndoa: Mfano wa Matumizi ya Semi Katika Jamii ya Wamasaaba Nchini Uganda”. East African Journal of Swahili Studies, Vol. 2, no. 2, June 2020, pp. 2331, doi:10.37284/eajss.2.2.169.

\section{UTANGULIZI}

Kila jamii kote ulimwenguni huwa na lugha. Lugha hizo hutofautiana kulingana na tamaduni za makabila mbalimbali. Kazi kuu ya lugha ni kuwezesha watu kuwasiliana. Mawasiliano huwa yanapelekea mambo mbalimbali kutekelezwa. Jambo mojawapo linalotekelezwa na lugha ni lile la kuelekeza mabinti katika maisha ya ndoa. Lugha kadhaa wa kadhaa huwa na vipengele mbalimbali vya kufanya hivyo. Vipengele hivyo hujumlisha nyimbo za kiutamaduni, semi, methali, misemo, usimulizi wa hadithi, n.k. Hata hivyo, katika makala hii, mtafiti anazingatia semi ambazo huwa zinatumiwa katika jamii ya Wamasaaba kwa ajili ya kuelekeza mabinti katika masuala ya ndoa. Hii ni kwa sababu katika jamii mbalimbali, kuna namna ambavyo watu wanatumia mazungumzo ya kawaida kuwaelekeza mabinti zao katika mambo tofautitofauti. Kila jamii huwa na semi maalum ambazo hutumiwa katika shughuli hii. Wao hawaandai kikao maalum cha kufunzia mabinti zao mambo hayo. Mafunzo yenyewe huwa yanajitokeza kwa njia ya mazungumzo ya kawaida. Ni hapo ndipo tunaona lugha ikifanya kazi katika jamii. Kwa hiyo, katika kazi hii, mtafiti anaeleza jinsi Wamasaaba wanavyotumia mazungumzo ya kawaida kufunza mabinti zao masuala ya ndoa. Ni kweli kwamba jamii mbalimbali zina semi kemkemu ambazo hutumiwa katika kazi hii. Hata hivyo, mtafiti anaangazia jamii ya Wamasaaba nchini Uganda. Anafanya hivyo kwa kueleza jinsi wazazi hutumia lugha kuwaandaa mabinti zao katika masuala ya ndoa. Matumizi ya lugha kwa njia hiyo hufanya wasichana wafikie umri wa kuolewa kama wanajua cha kufanya. Mtafiti anawasilisha semi mbalimbali zinazotumiwa na Wamasaaba, anazifasiri semi hizo katika lugha ya Kiswahili na anafafanua maana za semi mbalimbali.

\section{SUALA LA UTAFITI}

Kulingana na mtafiti, kiutamaduni, Waafrika walielekeza mabinti zao katika masuala ya ndoa kwa kutumia lugha. Jamii mbalimbali zilikuwa na semi maalumu ambazo walizitumia kuelekeza mabinti zao kuhusu maisha ya ndoa. Hii ilikuwa elimu jamii. Walifanya hivyo kwa sababu Waafrika walithamini ndoa jinsi ilivyokuwa ikiwaletea utajiri kutokana na mahari yaliyokuwa yakitolewa kwa wazazi. Kwa hiyo, wazazi walifurahia kuona ndoa za mabinti zao zinadumu. Kutokana na elimu jamii, wasichana walifikia umri wa kuolewa kama wamebobea katika masuala ya ndoa. Lakini wasichana wengi wa sasa huwa wanahitimu shule bila kumakinika katika masuala ya ndoa. Kwa wasichana hao, ndoa zao huwa zinavunjika mapema sana baada ya kuolewa. Hii ni kwa sababu lugha za mama huwa zinapuuzwa. Umuhimu wa lugha hizo za Kiafrika hauonekani tena. Kwa hiyo, watoto huwa wanakosa mambo mengi sana ambayo 
yalikuwa yakiwasilishwa kwao kwa kutumia lugha za mama. Jambo hili lilimpelekea mtafiti kuendeleza utafiti huu ili aeleze jinsi lugha za Kiafrika zinavyotumika na wazazi kuelekeza mabinti zao katika mambo ya ndoa. Mtafiti alirejelea jamii ya Wamasaaba nchini Uganda.

\section{MBINU ZA UTAFITI}

Katika kazi hii, mtafiti alitumia mbinu ya mahojiano. Kwa kutumia mbinu hii, mtafiti alizuru nyanjani halafu akakutana na wahojiwa wake. Kabla ya mahojiano, mtafiti alieleza wahojiwa wake sababu ya utafiti wake. Baada ya kufanya hivyo, alianza kuendeleza mahojiano na wahojiwa hao. Wahojiwa walijumlisha wanawake 50 na wanaume 50. Mtafiti alihakikisha kuwa kuna idadi sawa ya wanawake na wanaume kutokana na suala la usawa wa kijinsia. Wahojiwa hawa waliteuliwa kwa kutumia mbinu ya sharabera. Kwa kutumia mbinu hii, mtafiti aliendeleza mazungumzo na wahojiwa wake kama alivyokutana nao. Hakutumia utaratibu maalumu kuwateua wahojiwa hao. Mtafiti alikuwa na mwongozo wa maswali ambayo aliyatumia katika mahojiano. Mtafiti alitumia mwongozo ili asije akasahau jambo lolote muhimu.

\section{MAPITIO YA MAANDISHI}

Kwa mujibu wa Fitzwater (2020), kuna mambo 38 ya kujenga ndoa. Miongoni mwa mambo hayo: mwanamke akigundua jambo linalomkasirisha mumewe, akome kulifanya. Asimwambie mumewe kutekeleza matakwa yake yote kwa sababu ni Mungu peke yake ndiye anaweza kufanya hivyo. Endapo mumewe atakosa kuelewa hisia zake, amwambie. Kama hajafahamu kile anachokifikiria, amwambie. Hajafahamu kwa nini hana furaha, amwambie. Hakuacha mambo anayoyapenda kama vile michezo alipomuoa, amwache aendelee nayo. Asimzuie kufanya mambo anayoyapenda maishani mwake, kwa sababu Mungu peke yake ndiye anaweza kumbadilisha mtu. Kwa hiyo, ampe nafasi. Asiwaambie marafiki zake mambo yanayomuhusu mumeo.

Licha ya mapungufu yake, yafaa awaambie marafiki zake jinsi alivyo na mume mzuri. Amgeukie Bwana Yesu. Ni yeye ndiye atampa furaha na kutekeleza matakwa yake yote. Amuombee mume wake kila wakati. Ajifunze kuwa mpishi mzuri. Na kama hawezi kufanya hivyo, ampumbaze wakiwa kitandani. Asimwache amalize siku kadhaa bila ngono. Kama hayuko katika hali ya ngono, afanye juhudi za kumsisimua. Mungu ni mwaminifu katika masuala ya pesa, ya kiroho na kutoa msamaha. Lakini ni mwanifu pia katika mambo ya ndoa. Anaweza kumwomba ampe usaidizi na maarifa humo chumbani jinsi anavyomwomba kokote kwingine. Amche Mungu na atamfanyia maajabu mume wake. Haya ni baadhi tu ya mawaidha anayoyapendekeza Fitzwater (2020).

Kulingana na YourTango (2018), mapenzi ni jambo la kujitolea wala si la kuhisi. Mwandishi wa makala haya anatoa mawaidha 23 ya kuzingatiwa na wanawake katika maisha ya ndoa. Kulingana na mwandishi huyu, mawaidha hayo ni kujizatiti kumpenda mwenzako hata kama mapenzi yenyewe yanaonekana kuwa yamefifia. Kila unapopigiwa simu na mwenzako, ipokee na umjibu. Kutofanya hivyo huzusha tashwishi na kuleta migogoro katika ndoa. Hakikisha kuwa mna wakati wa kutosha mkiwa pamoja. Jambo la kuwa pamoja ni muhimu sana katika maisha ya ndoa. Hii ni kwa sababu kila mmoja anamhitaji mwenzake. Karibisha marafiki wataoijenga ndoa yako wala hao ambao wataivunja. Tabasamu na ikiwezekana cheka na mumeo hata kama hali yenyewe ni ngumu. Mnapojadili jambo tatanishi, kila mmoja wenu asijaribu kuibuka mshindi. Kama ni kushinda mfanye hivyo pamoja. Hali kadhalika, kama ni kushindwa, mfanye hivyo pamoja. Hii ni kwa sababu ndoa iliyo imara huwa haina watu wawili wenye nguvu wanaoshindana. Katika ndoa hakuna aliye na nguvu kumshinda mwenzake. Lipe uzito jambo linalotokea katika chumba chenu mnamolala. Kujenga ndoa imara si ngono peke yake. Kuna mambo ya ziada yanayostahili kutendeka ili kujenga ndoa imara. Kumbuka ndoa si 50/50 lakini talaka ni 50/50. Ndoa yafaa iwe 100/100. Mpe mumeo au mkeo kilicho bora wala si kile kinachobakia baada ya kuwapa watu wengine vilivyo bora. Jifunze kutoka kwa watu wengine lakini usije ukafikiria kulinganisha maisha yako au ndoa yako na ya mtu mwingine. Ukimkosea mwenzako, kubali kosa lako na uombe msamaha mara moja. Ukivunjika moyo na kosa la mwenzako, msamehe papo hapo na umwambie "nakupenda, nimekusamehe." Vumiliana katika mambo 
mbalimbali yanayojitokeza katika ndoa yenu. Usiwe na haraka ya kumhukumu mume wako.

Kwa mujibu wa Papandrea (2012), kuna kanuni kumi ambazo zinastahili kuzingatiwa na wanandoa. Naye anasema kanuni hizi ni: usije ukasema mabaya ya wazazi wa mumeo. Ni wazi kuwa anaweza kuchukiwa na wazazi wake lakini hatakubali mtu yeyote kuzungumza juu yao. Hii ndio sababu unastahili kuwa makini unapozungumza juu ya wakwe zako. Mwambie mumeo wapenzi wako wa zamani. Hili litakusaidia kujitetea ukitumiwa jumbe za ghafla kutoka kwao. Yahifadhi mawaidha unayopewa na watu bila wewe kuwaomba wafanye hivyo. Katika hali hiyo, toa mchango wako, sikiliza lakini usithubutu kusema "ninajua kilicho bora." Usiwe mbele ya kila kitu. Usifanye kila kitu kwa kufikiria kuwa mwenzako hafanyi vizuri kama wewe. Usifufue mambo yaliyopita. Ukiudhika na jambo, sema. Mwambie mumeo kuwa unataka mambo yawe hivi badala ya kunyamaza. Usitume mitandaoni mawazo au picha za kibinafsi. Afikiana kama wanandoa kuhusu mambo ya kutuma mitandaoni. Usimalize muda mrefu mtandaoni. Mwenzako anaweza kufikiria kuwa yeye si muhimu kwako. Usitumie msamiati "talaka." Hata kama ugomvi umepamba moto, usije ukatishia kubeba mizigo yako. Hili ni jambo hatari sana katika ndoa. Fanya mumeo kuwa mtu wa kwanza katika maisha yako. Jilinde na mambo ya watu wa nje ambayo yanaweza kuharibu ndoa yako.

Kwa mujibu wa Pillemer (2015), kabla ya ndoa, wachumba wanastahili kufikiria jinsi watu walivyofikiria nyakati za kale. Kwanza, msichana afikirie kama mume wake mtarajiwa atakuwa na uwezo kumpa mahitaji yake. Mtaalamu huyu anasema kuwa ndoa ni mapenzi lakini huathiriwa na hali ya kiuchumi. Kwa hiyo, ni lazima msichana aangalie hilo. Aidha, msichana anastahili kujiuliza kama watu wengine wanampenda mume wake mtarajiwa. Pillemer (2015) anasema kuwa msichana hawezi akafanya uamuzi peke yake. Anapendekeza msichana awasikilize wazazi wake na marafiki aone wanasema nini kuhusu huyo mchumba wake. Wanampenda? Anamdanganya? Amejitolea kikamilifu kumuoa? Hakikisha kuwa mipango ya mumeo mtarajiwa kuhusu ndoa yanaafikiana na yako. Malengo yako yaende sambamba na yake.
Kwa mujibu wa Warwicker (2020), ndoa ni kitendo cha kudumu. Kwa hiyo, ni muhimu kuelewa ni nini kinachoweza kufanya ndoa yako kuwa imara na kukupa uwezo wa kukabiliana na mambo tatanishi unayokumbana nayo. Mtaalamu huyu anapendekeza mambo kadhaa ya kuzingatiwa miongoni mwa mambo hayo ni kusameheana. Anasema hili ni jambo muhimu sana katika ndoa. Anasema kosa likitokea, samehe mara moja. Usiseme umesamehe halafu ubakishe jambo moyoni. Jambo jingine linalopendekezwa na mtaalamu huyu ni lile la kusikilizana. Anasema yafaa kila mtu amsikilize mwenzake. Huenda jambo analo mwenzako si zuri lakini msikilize. Kulingana na Warwicker (2020), wanandoa wanastahili kuwa marafiki, wafanye kazi pamoja na wapate muda wa kutembea pamoja. Mtaalamu huyu anasema usijaribu kufanya maajabu katika mwaka wa kwanza wa ndoa yenu, kama vile kubadilisha nyumba. Kilicho muhimu ni kukubali kuwa mmeanza maisha ya ndoa. Wanandoa wanastahili kuaminiana. Msifichane mambo fulani. Kufanya hivyo ni hatari kwa ndoa yenu.

Lowe (2019) anaorodhesha semi mbalimbali kuhusu mapenzi na ndoa. Baadhi ya semi ambazo zimeorodheshwa ni hizi zifuatazo: penye mapenzi pana uhai; mapenzi hayajui mipaka, yanaruka vizuizi, yanapenyeza kuta na kufika mwisho wa safari yakiwa yamejaa matumaini; ndoa ya kudumu huhitaji wanandoa kuendelea kuibua mapenzi upya baina yao mara kadhaa; kupendwa sana na mtu kunakupa nguvu ilhali wewe kupenda mtu sana kunakupa hamasa. Kwa kutazama semi ambazo zimetajwa, tunaona kwamba zinawasilisha mawaidha ya kujenga wanandoa. Hata hivyo, semi zenyewe hazijahusishwa na jamii yoyote ile. Kwa hiyo, kama Waafrika ni muhimu kuweka bayana zile semi za Kiafrika na kuonyesha jinsi zinavyofanya kazi katika jamii zetu.

Kwa mujibu wa Aldrich (2020), siku ambayo mtu hukubali mnapofunga ndoa ni miongoni mwa nyakati maalumu katika maisha. Haijalishi kama umeolewa majuma machache yaliyopita au mmemaliza muda mrefu katika ndoa au umo katika ndoto ya kuoa. Semi unazozisikia zinakukumbusha juu ya uamuzi muhimu baina ya watu wawili. Miongoni mwa semi, anataja semi kama vile "ndoa zenye furaha huanza na kuoa watu tunaowapenda na ndoa hizo hunawiri tukipenda wale tunaowaoa, 
ndoa inayodumu huhitaji kuweka upya mapenzi mara kadhaa na yule mtu mmoja.

Guttmann (2020) naye anataja semi zifuatazo: Naipenda miguu yako iliyotembea na kunitafuta; ni afadhali kuyeyuka kutokana na mapenzi ya baadhi ya watu; wewe ni jua langu, mwezi na nyota zangu; kitu cha kushikilia maishani ni mumeo au mkeo, mwishowe mapenzi unayotwaa ni sawa na mapenzi unayotengeneza. Khurana (2019) anataja semi kama vile ni jambo zuri kwa wapendanao kulalia mto mmoja; kwa dunia mnaweza kuwa mtu mmoja bali kwa mtu mmoja mnaweza kuwa dunia; hakuna jambo zuri kuliko ndoa yenye furaha; wapendanao hawakutani mahali fulani - kila mmoja huwa ndani ya mwenzake; neno moja linatuletea uhuru maishani mwetu na neno hilo ni upendo.

\section{MTAZAMO WA MTAFITI KUHUSU MAPITIO YA MAANDISHI}

Baada ya kudurusu maandiko mbalimbali, mtafiti aligundua kwamba kuna wataalamu wengi sana ambao wameandika kuhusu mawaidha yanayofaa kutolewa na wazazi pamoja na marafiki kwa wasichana wanaojiandaa kwenda kuolewa. Haya ni mawaidha yanayotolewa rasmi kwa msichana ambaye anajiandaa kuolewa. Ni mawaidha mazuri na ambayo yanaweza kumjenga msichana katika masuala ya ndoa. Hata hivyo, waandishi hao hawajahusisha maelezo yao na jamii mahususi. Maelezo yenyewe ni ya jumla. Zaidi ya hilo, maelezo yenyewe yanalenga jamii za magharibi. Wao hawajazungumzia jamii za Kiafrika na kuonyesha jinsi wazazi wanavyotumia mazungumzo ya kawaida kuwafunza mabinti zao masuala ya ndoa. Jambo hili linaacha mwanya ambao mtafiti ameuziba katika kazi hii. Mtafiti ameuziba mwanya huo kwa kueleza jinsi Wamasaaba nchini Uganda wanavyotumia lugha kuelekeza mabinti zao katika maisha ya ndoa. Huu ni mchakato unaotekelezwa kabla ya wasichana hawajafikia umri wa kuolewa. Yaani, maelekezo hayo ni sehemu ya maisha ya watoto. Wazazi huwa hawasubiri wakati wa ndoa kabla kueleza mabinti zao. Wao huwa wanafanya hivyo watoto wanapokuwa wakikua.

\section{MATOKEO YA UTAFITI}

\section{Jedwali 1: Semi Zinazotumiwa na Wamasaaba Kuelekeza Wasichana Katika Masuala ya Ndoa}

\begin{tabular}{ll}
\hline Semi Katika Lugha Asili & Tafsiri Katika Kiswahili \\
\hline Umusetsa numukhasi bamenyanga bakulo. & $\begin{array}{l}\text { Mke na mume huishi kwa kufanyiana } \\
\text { mizaha. }\end{array}$ \\
\hline Umusetsa nga wakhomile basili bowo ubabolelama ta. & Mumeo akiwatusi wazazi wako usiwaambie. \\
\hline Umusetsa alurama wakhulekha mubulili ta. & Mumeo hatoki akakuacha kitandani. \\
\hline Umusetsa nga alomana usila. & Mumeo akigombana unanyamaza. \\
\hline Umusetsa umukyungila enda. & Mumeo unamchungia tumbo. \\
\hline Umukhasi arusama bibyama. & Mke hastahili kutoa sili za ndoa \\
\hline Umukhasi alurama ango. & $\begin{array}{l}\text { Mke hatoki nyumbani na kwenda } \\
\text { kuzururazurura. }\end{array}$ \\
\hline Umukhasi alyama ingokho. & Mke havai suruali. \\
\hline Umukhasi unywesa ikyembe. & Mke hali nyama ya kuku. \\
\hline Umukhasi ulinda babana bowo. & Mke hushika jembe kwa ukakamavu. \\
\hline Umukhasi ukholama lukambo. & Mke mzuri analinda watoto wake. \\
\hline Umukhasi utambama khanyinyi ango. & Mke hashiriki katika masengenyo. \\
\hline Umukhasi utambaa lusisi & Mke hakosi mboga nyumbani. \\
\hline
\end{tabular}

Katika Jedwali 1, mtafiti anawasilisha semi mbalimbali ambazo zinasemekana kuwa zinatumiwa na Wamasaaba kuelekeza wasichana wao katika masuala ya ndoa. Semi hizo zimeandikwa katika lugha asili ambayo ni Kimasaaba halafu zikafasiriwa katika lugha ya Kiswahili. Tukiangalia Jedwali 1, tunaona kuwa katika upande wa kushoto kuna namba 
zinazoonyesha semi mbalimbali ambazo zimetajwa. Katika sehemu ya katikati, kuna semi zenyewe zikiwa zimeandikwa katika lugha asili. Katika upande wa kulia, kuna tafsiri za semi ambazo zimetajwa. Mtafiti alifasiri semi hizo katika lugha ya Kiswahili ili kufanya wasomaji wake wapate kuzielewa.

\section{Jedwali 2: Mara Tokezi ya Kila Usemi Unaotajwa}

\begin{tabular}{ll}
\hline Semi Katika Lugha Asili & Mara Tokezi \\
\hline Umusetsa numukhasi bamenyanga bakulo. & 94 \\
Umusetsa nga wakhomile basaali bowo ubabolelama ta. & 77 \\
Umusetsa alurama wakhulekha mubulili ta. & 98 \\
Umusetsa nga alomana usila. & 95 \\
Umusetsa umukyungila inda. & 66 \\
Umukhasi arusama bibyama. & 93 \\
Umukhasi alurama ango. & 67 \\
Umukhasi etuusa ango. & 56 \\
Umukhasi alyama ingokho. & 72 \\
Umukhasi unywesa ikyembe. & 84 \\
Umukhasi ulinda babana bowo. & 90 \\
Umukhasi ukholama lukambo. & 83 \\
Umukhasi utambama khanyinyi ango. & \\
Umukhasi atambamaa lusisi & 88 \\
\hline
\end{tabular}

Tukirejelea Jedwali 2 tunaona kuwa miongoni mwa semi zinazosemekana kuwa zinatumiwa na Wamasaaba kuelekeza mabinti zao katika masuala ya ndoa ni ule usemi usemao kwamba "mke na mume huishi kama Bakulo;" hoja hii ilirudiwa mara 94 (94\%). Neno Bakulo ambalo ni la Kimasaaba linamaanisha watu wawili wenye uhusiano maalumu unaomruhusu kila mmoja kumwambia mwenzake mambo mabaya kimzaha na hakuna kukasirika au kuenda mahakamani kuwasilisha malalamishi. Kwa kutumia usemi huu, Wamasaaba wanamaanisha kuwa katika ndoa kuna kukwaruzana baina ya mke na mume lakini baada ya kufanya hivyo wanastahili kurudi pamoja. Mume hastahili kumfukuza mkewe eti kwa sababu amemtusi au mke kufunga virago na kuondoka eti mumewe amemtusi. Wamasaaba huwa wakitumia usemi huu kila wanapozungumza na watoto wao wa kike ambao wanakaribia kuvunja ungo. Wao wanafanya hivyo kwa ajili ya kuwafanya wawe wavumilivu baada ya kuolewa. Hawataki mabinti zao wawe wakikimbilia makwao kila mara wakilalamika juu ya waume zao.

Jedwali 2 vile vile linaonyesha usemi mwingine ambao hutumiwa na Wamasaaba kuelekeza mabinti zao katika masuala ya ndoa ni ule usemao kwamba "mume akiwatusi wazazi wa mkewe, mkewe hastahili kuwaambia." Wamasaaba wanatumia usemi huu kwa sababu wanajua kwamba ndoa zina vituko. Wanandoa wakianza ugomvi, kila mmoja anaweza kusema jambo lolote mkiwemo kuwatusi wakwe zao. Hata hivyo, mke hastahili kuwaambia wazazi wake kwamba mumewe amewatusi. Hii ni kwa sababu jambo hilo linaweza kuvunja ndoa yao. Hoja hii ilisisitizwa na wahojiwa 77 (77\%).

Wamasaaba hutumia usemi usemao "mume hatoki akamwacha mkewe kitandani akilala" kuwafundisha mabinti zao kufanya kazi. Kulingana na Wamasaaba, mke anayebakia kitandani akilala ni mzembe. Na ndoa za wanawake wazembe mara nyingi huvunjika. Kwa hiyo, Wamasaaba hutumia usemi huu kwa ajili ya kufundisha mabinti zao kuwa wakiolewa, hawastahili kubakia vitandani wakilala huku waume zao wakitoka kwenda mashambani kulima. Kwa Mmasaaba wa jadi, mke ndiye anatangulia kwenda shambani. Mumewe anaweza kumfuata baadaye au asimfuate. Mume kutomfuata mkewe shambani si suala. Hoja hii iliangaziwa na wahojiwa 98 (98\%) (Jedwali 2).

Hali kadhalika, Jedwali 2 linaonyesha kuwa Wamasaaba hutumia usemi usemao kuwa "mume akianza ugomvi, mkewe anapaswa kunyamaza." Wamasaaba wanatumia usemi huu kwa sababu 
wanajua kwamba ndoa nyingi huwa zinavunjika kutokana na ugomvi. Wao hutumia usemi huu ili kuwafunza mabinti zao kudumisha amani katika familia zao. Wanatumia usemi huu kwa kujua kwamba mume akigombana halafu mkewe anyamaze, bila shaka atakoma kufanya hivyo. Hii ni kwa sababu mtu mjinga ndiye anaendelea kugombana na mtu asiyemjibu. Lakini mume akianza ugomvi halafu mke naye aanze, basi, wao watapigana. $\mathrm{Na}$ wakipigana, wataumizana na mwishowe ndoa yao itavunjika. Hili ni jambo ambalo halistahili kutokea katika jamii za Kiafrika. Wamasaaba na Waafrika kwa kijumla huwa wanafurahia ndoa zinazodumu. Hii ndiyo sababu wakawa wakitumia semi hizi katika mazungumzo ya kawaida ili kufanya mabinti zao wakue wakiwa wamemakinika katika masuala ya ndoa. Hoja hii ilisisitizwa na wahojiwa 95 (95\%) (Jedwali 2).

Wahojiwa $66 \quad(66 \%)$ walirejelea kwamza Wamasaaba hutumia usemi wa "mke anapaswa kumchungia mume tumbo" kuwafundisha mabinti zao kuwapikia waume zao chakula (Jedwali 2). Kwa Mmasaaba wa jadi, mume hawezi akatoka nyumbani bila kula. Jambo ambalo mke anapaswa kumfanyia mumewe kama hajatoka nyumbani ni kumpa chakula. Hata kama haendi popote, ana haja ya kula. Mke hastahili kumwacha mumewe akashinda njaa. Yafaa ahakikishe kuwa ana chakula cha kumpa mumewe hata kama kuna watoto ambao pia wanataka kula. Ni wajibu wa baba kuchukua kile ambacho amepewa awape watoto wake kidogo kidogo. Wamasaaba huwafunza mabinti zao namna hii kwa sababu wanajua kwamba mume akishinda njaa, anaweza kuoa mke mwingine au kumfukuza yule aliye naye nyumbani.

Asilimia 93 ya wahojiwa walinema kwamba Wamasaaba hufunza mabinti zao kutotoa siri za maboma yao kupitia usemi huu: "mke hatoi siri za nyumbani" (Jedwali 2). Baadhi ya wanaume huwa na mapungufu yao na hawataki yajulikane. Kwa hiyo, mwanamke akitaka kubakia katika ndoa yake anapaswa kuhifadhi siri hizo. Kwa mfano, mumewe anaweza kuwa mchawi au anakojoa kitandani. Mke hapaswi kumtangaza. Kutangaza mambo kama haya huzusha ugomvi, vita na matokeo yake ni ndoa kuvunjika.

Kulingana na Jedwali 2, Wamasaaba huelekeza mabinti zao katika masuala ya ndoa kupitia "mke hatoki nyumbani." Kwa Mmasaaba wa jadi, mke hastahili kutoka nyumbani bila kuwa na sababu maalumu. Kwao, mume ndiye anapaswa kutoka kuenda kutafuta riziki. Akirejea nyakati za jioni, mkewe ndiye anastahili kumpokea mzigo ambao amemletea. Kwa hiyo, si vizuri mwanaume kurudi halafu apate kwamba mkewe pia ametoka kwenda kuzururazurura. Jambo hili pia hupelekea ndoa kuvunjika. Kutokana na hili, Wamasaaba huwa wakielekeza mabinti zao kabla ya kuolewa kwa kutumia usemi kwamba si vizuri mwanamke kutoka nyumbani kwenda matembezini. Hoja hii ilirejelewa na wahojiwa 67 (67\%) (Jedwali 2).

Wahojiwa 56\% watazama kuwa, Wamasaaba hutumia pia usemi usemao "mke hujulikana kwa moshi" (Jedwali 2). Hii inamaanisha kuwa yeye ni mwanamke wa kupikia watu, yaani si mchoyo. Hii ni kwa sababu makapera hurauka asubuhi na kurejea usiku. Kwa hiyo, si rahisi kuona moshi pale kwao. Lakini akioa, kila mara moshi huonekana pale kwake. Kuwepo kwa moshi kuna maana kuwa mke anapika. Jambo hili linamaanisha kuwa mwanamke anastahili kuwasha moto hata kama ni kuchemsha maji tu. Kutokana na jambo hili, Wamasaaba huwa wanafunza mabinti zao kwa kutumia usemi kuwa mke anajulikana kwa moshi.

Kwa kuzingatia data zilizopo katika Jedwali 2, inabainika wazi kuwa Wamasaaba huelekeza mabinti zao kuhusu masuala ya ndoa kwa kutumia usemi "mke hali nyama ya kuku." Kama ilivyo na watu wengi, nyama ya kuku ni nzuri kuliko aina zingine za nyama. Karibu kila mtu huwa anapenda kula nyama ya kuku. Kwenye karamu mtu akikosa kupewa nyama ya kuku hugombana hata kama amepewa nyama ya ng'ombe. Kwa hiyo, ili kuepukana na mizozo inayotokana na mke kula kipande cha kuku, Wamasaaba wa jadi, walikuja na usemi kwamba "mwanamke hali nyama ya kuku." Usemi huu ulidhamiria kuwazuia kabisa wanawake kula nyama hiyo. Na kusema ukweli, si rahisi wanawake wakongwe kula nyama ya kuku katika jamii hii. Kwao, kula nyama hiyo ni mwiko. Ni wanawake wa kisasa ndio wamevunja mwiko huo na kuanza kula. Tukitazama Jedwali 2, tunaona kuwa hoja hiyo ilijitokeza mara 72 (72\%).

Hali kadhalika, usemi mwingine unaotumiwa na Wamasaaba kufundisha mabinti zao kuhusu mambo ya ndoa ni ule usemao kwamba 
"mwanamke hushika jembe kwa nguvu." Wamasaaba hutumia usemi huu kufundisha mabinti zao kufanya kazi. Hii ni kwa sababu, mwanamke yeyote ambaye hana kazi ya serikali hutegemea jembe kulisha familia yake. Mwanamke anayefanya kazi kwa bidii husifiwa na jamii yake huku yule anayezembea kazi hukashifiwa. Mwanamke anayezembea kazi huletea wazazi wake aibu. Hii ndiyo sababu Wamasaaba wakatumia usemi huu kuwa "mwanamke hushika jembe kwa nguvu." Hoja ilirudiwa mara 84 (84\%) kulingana na jedwali Na. 2.

Wamasaaba hutumia usemi "mke hulinda watoto wake" kuelekeza mabinti zao umuhimu wa ulezi. Wao hutumia usemi huu kwa sababu kuna baadhi ya wanawake ambao wanaacha watoto wao ovyo ovyo na uenda zao. Hawajui kupikia watoto wao, jambo ambalo linawafanya waende kula kwa majirani. Jambo hili huharibu uhusiano na huleta ugomvi na majirani hao. Mwanamume akiona kwamba mkewe hawezi kuwapikia watoto wake, anaweza kuamua kumuoa mke mwingine au hata kumfukuza yule aliyenaye nyumbani. Kwa hiyo, ili kuwaepusha mabinti zao na jambo hili, Wamasaaba huwafunza kwa kutumia usemi huu kuwa "mke hulinda watoto wake." Usemi huu huwafanya wakue wakijua kwamba si vizuri mwanamke kuacha watoto wake ovyo ovyo. Kulingana na Jedwali 2, hoja hii lirejelewa ana wahojiwa 90.

Vile vile, Jedwali 2 linaonyesha kuwa Wamasaaba huwa wanaelekeza mabinti zao katika mambo ya ndoa kwa kutumia usemi kwamba "mwanamke hashiriki katika masengenyo." Kulingana na Wamasaaba, masengenyo husababisha ugomvi. Ugomvi huleta uadui na mambo mengine ambayo si mazuri. Kwa hiyo, mwanaume akiona kwamba mkewe huwa anagombana na majirani anaweza kumpa talaka. Kutokana na jambo hili, wazazi walikuja na usemi kwamba "mwanamke hashiriki katika masengenyo." Walitumia usemi huu kuwaelekeza mabinti zao ili wasije wakafanya hivyo baada ya kuolewa.

Usemi "mwanamke hakosi konde la mboga nyumbani kwake" pia hutumiwa kutoa mawaidha kwa wasichana. Usemi ulisisitizwa na wahojiwa 89 (89\%). Wamasaaba hutumia usemi huu ili kufundisha mabinti zao kuwa mke wa mtu lazima awe na konde la mboga nyumbani kwake.
Waliamua kutumia usemi huu kwa sababu kwa wanawake wengine, mgeni akija nyumbani kama mumewe hayuko, hawezi akampikia chakula akidai kwamba mumewe hayuko na kwamba hajamwachia pesa za mboga. Kwa Mmasaaba wa jadi, jambo hili halifai. Kwao, mwanamke anastahili kuwa na kijishamba cha mboga karibu na nyumba yao ili mgeni akija, anaenda tu pale na kuzichuna halafu ampikie mgeni chakula bila kumsubiri mumewe.

Mwishowe, Wamasaaba hutumia usemi "mwanamke hakosi jiwe la kusagia wimbi." Kulingana na Jedwali 2, hoja hii ilirudiwa mara 88 (88\%). Jiwe hili ni kifaa muhimu kwa Mmasaaba wa jadi. Kulingana na Wamasaaba, jiwe hili si kifaa cha kuombaomba. Kila familia inastahili kuwa nalo. Kwa hiyo, kuombaomba jiwe hili kunaweza kuzusha ugomvi na majirani. Ugomvi na majirani unaweza pia kumfanya mwanaume amfukuze mkewe. Kutokana na jambo hili, Wamasaaba wanatumia usemi huu ili kuhamasisha mabinti zao wasiwe wa kuombaomba jiwe hilo baada ya kuolewa. Hapa tunaona kuwa usemi huu ulidhamiria kuwapa mawaidha mabinti zao ya kuepuka na mizozo na majirani.

\section{HITIMISHO}

Kwa kuzingatia data ambazo zimewasilishwa na kujadiliwa katika makala hii, inabainika wazi kuwa lugha za Kiafrika huwa zinafanya kazi kubwa sana ya kuelimisha wasichana mambo ya ndoa. $\mathrm{Na}$ si mambo ya ndoa peke yake bali mambo mengine pia yenye uhusiano na maisha ya binadamu. Hii inamaanisha kuwa lugha ndogo ndogo za Kiafrika zina machango mkubwa sana katika maisha ya vijana. Kwa hiyo, haifai watu kuzipuuza lugha hizo ndogo ndogo. Yafaa lugha hizo zikuzwe badala ya kuangamizwa. Kutokana na umuhimu huu wa lugha zetu, viongozi katika jamii mbalimbali za Kiafrika wanastahili kuweka mikakati ya kukuza lugha hizi katika maeneo yao ya utawala. Wanastahili kufanya juhudi za kukuza na kuendeleza lugha hizo. Serikali kuu za nchi mbalimbali za Kiafrika zinapaswa kuweka sera madhubuti kuhakikisha kuwa lugha za Kiafrika zinakuzwa na kuendelezwa. Hii ni kwa sababu zina mambo mengi mazuri ambayo hayajajulikana kwa ulimwengu. Kwa hiyo, ni wajibu wa wanafasihi na wanaisimu kuzitafiti lugha zetu ndogo ndogo katika 
Jumuiya ya Afrika Mashariki na hata bara zima la Afrika ili kuweka wazi mambo mazuri ambayo hayajajulikana.

\section{MAREJELEO}

Aldrich J. (2020, Apr 24). 29 Wedding Quotes to Take You Back to the Day You Said 'I Do.' Retrieved from Country Living, available at https://www.countryliving.com/life/g2740922 2/wedding-quotes/.

YourTango. (2018, Sept 2). 23 Pieces of the Best Marriage Advice EVER (Collected Over 13 Years). Retrieved from Brides, available at https://www.brides.com/story/pieces-of-bestmarriage-advice-ever-collected-over-years.

Fitzwater, C. (2020). The Best 38 Bits of Marriage Advice A Woman Could Get. Retrieved from Club31 Women, available at https://club31women.com/38-best-bits-ofmarriage-advice-a-woman-could-get/.

Guttmann, A. (2020). 90 Short and Sweet Love Quotes that Will Speak Volumes at Your Wedding. Retrieved from Martha Stewart Wedding, available at https://www.marthastewartweddings.com/395 782/short-sweet-love-quotes-your-wedding.

Pillemer, K. (2015, Feb 1). The Advice that Older Women Have for the Young on Love. Retrieved from The World, available from https://www.pri.org/stories/2015-02-

01/advice-older-women-have-young-lovemarriage.

Khurana, S. (2019, Jun 5). 19 Famous Quotes for Inspirational Marriage Wishes. Retrieved from ThoughtCo, available at https://www.thoughtco.com/marriage-wishesand-quotes-2832678.

Lowe L. (2019, Dec 13). 100 Inspiring Quotes on Love and Marriage. Retired from Parade, available

at https://parade.com/391231/lindsaylowe/inspiri ng-quotes-love-marriage/ .

Papandrea, D. (2012, Oct 11). 10 Unspoken Marriage Rules You Must Follow. Retrieved from: WomansDay, available at https://www.womansday.com/relationships/da ting-marriage/advice/a6718/marriage-rules/.

Warwicker, S. (2020). Real Bride's Words of Wisdom for a Happy Marriage. Retrieved from You and Your Wedding, available at https://www.youandyourwedding.co.uk/ideasand-advice/wedding-budget/real-brideswords-of-wisdom-for-a-happy-marriage/. 